CORRIGENDUM

doi:10.1038/nature04475

\section{Exogenous and endogenous glycolipid antigens activate NKT cells during microbial infections}

Jochen Mattner, Kristin L. DeBord, Nahed Ismail, Randal D. Goff, Carlos Cantu III, Dapeng Zhou, Pierre Saint-Mezard, Vivien Wang, Ying Gao, Ning Yin, Kasper Hoebe, Olaf Schneewind,

David Walker, Bruce Beutler, Luc Teyton, Paul B. Savage

\& Albert Bendelac

Nature 434, 525-529 (2005)

Figure 3 of this Letter contains an inadvertently duplicated panel: the PBS 30 panel is identical to the $\alpha$ GalCer panel (top right). The corrected panels are shown here. Our results and conclusions are unaffected by this oversight.
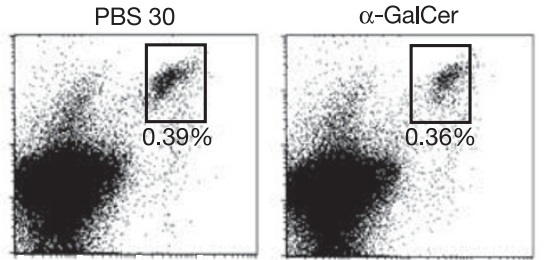

\section{CORRIGENDUM}

\section{doi:10.1038/nature04484}

\section{Genome sequencing in microfabricated high-density picolitre reactors}

Marcel Margulies, Michael Egholm, William E. Altman, Said Attiya, Joel S. Bader, Lisa A. Bemben, Jan Berka, Michael S. Braverman, Yi-Ju Chen, Zhoutao Chen, Scott B. Dewell, Alex de Winter, James Drake, Lei Du, Joseph M. Fierro, Robin Forte,

Xavier V. Gomes, Brian C. Goodwin, Wen He, Scott Helgesen, Chun He Ho, Steve Hutchinson, Gerard P. Irzyk,

Szilveszter C. Jando, Maria L. I. Alenquer, Thomas P. Jarvie, Kshama B. Jirage, Jong-Bum Kim, James R. Knight, Janna R. Lanza, John H. Leamon, William L. Lee, Steven M. Lefkowitz, Ming Lei, Jing Li, Kenton L. Lohman, Hong Lu, Vinod B. Makhijani, Keith E. McDade, Michael P. McKenna, Eugene W. Myers, Elizabeth Nickerson, John R. Nobile, Ramona Plant, Bernard P. Puc, Michael Reifler, Michael T. Ronan, George T. Roth, Gary J. Sarkis, Jan Fredrik Simons, John W. Simpson, Maithreyan Srinivasan, Karrie R. Tartaro, Alexander Tomasz, Kari A. Vogt,

Greg A. Volkmer, Shally H. Wang, Yong Wang, Michael P. Weiner, David A. Willoughby, Pengguang Yu, Richard F. Begley

\& Jonathan M. Rothberg

Nature 437, 376-380 (2005)

The following were omitted from the original author listing: Alex de Winter, James Drake, Robin Forte, Steve Hutchinson, William L. Lee, Michael Reifler and David A. Willoughby. These names are included in the revised authorship shown here and either were or are at 454 Life Sciences Corporation, Branford, Connecticut 06405, USA.

\section{CORRIGENDUM}

\section{doi:10.1038/nature04572}

\section{Genomic sequence of the pathogenic and allergenic filamentous fungus Aspergillus fumigatus}

William C. Nierman, Arnab Pain, Michael J. Anderson, Jennifer R. Wortman, H. Stanley Kim, Javier Arroyo, Matthew Berriman, Keietsu Abe, David B. Archer, Clara Bermejo, Joan Bennett, Paul Bowyer, Dan Chen, Matthew Collins, Richard Coulsen, Robert Davies, Paul S. Dyer, Mark Farman, Nadia Fedorova, Natalie Fedorova, Tamara V. Feldblyum, Reinhard Fischer, Nigel Fosker, Audrey Fraser, Jose L. García, Maria J. García, Arlette Goble, Gustavo H. Goldman, Katsuya Gomi, Sam Griffith-Jones, Ryan Gwilliam, Brian Haas, Hubertus Haas, David Harris, H. Horiuchi, Jiaqi Huang, Sean Humphray, Javier Jiménez, Nancy Keller, Hoda Khouri, Katsuhiko Kitamoto, Tetsuo Kobayashi, Sven Konzack, Resham Kulkarni, Toshitaka Kumagai, Anne Lafon, Jean-Paul Latgé, Weixi Li, Angela Lord, Charles Lu, William H. Majoros, Gregory S. May, Bruce L. Miller, Yasmin Mohamoud, Maria Molina, Michel Monod, Isabelle Mouyna, Stephanie Mulligan, Lee Murphy, Susan O'Neil, Ian Paulsen, Miguel A. Peñalva, Mihaela Pertea, Claire Price, Bethan L. Pritchard, Michael A. Quail, Ester Rabbinowitsch, Neil Rawlins, Marie-Adele Rajandream, Utz Reichard, Hubert Renauld, Geoffrey D. Robson, Santiago Rodriguez de Córdoba, Jose M. Rodríguez-Peña, Catherine M. Ronning, Simon Rutter, Steven L. Salzberg, Miguel Sanchez, Juan C. Sánchez-Ferrero, David Saunders, Kathy Seeger, Rob Squares, Steven Squares, Michio Takeuchi, Fredj Tekaia, Geoffrey Turner, Carlos R. Vazquez de Aldana, Janice Weidman, Owen White, John Woodward, Jae-Hyuk Yu, Claire Fraser, James E. Galagan, Kiyoshi Asai, Masayuki Machida, Neil Hall, Bart Barrell \& David W. Denning

\section{Nature 438, 1151-1156 (2005)}

There are two errors in the author listings for this Letter: the surname of Anne Lafon was misspelt as 'Lafton' and the affiliation of Hiroyuki Horiuchi should have been number 16 (and not 15, as was published).

\section{ERRATUM}

\section{doi:10.1038/nature04476}

\section{Regulated cell-to-cell variation in a cell-fate decision system}

Alejandro Colman-Lerner, Andrew Gordon, Eduard Serra, Tina Chin, Orna Resnekov, Drew Endy, C. Gustavo Pesce \& Roger Brent

\section{Nature 437, 699-706 (2005)}

In Fig. $1 \mathrm{~b}$ of this Article, the $x$ axis of the right-hand plot should be labelled ' $\alpha$-Factor system output in each cell (CFP F.U. $\times 10^{6}$ )' and not 'ACT1 system output in each cell (CFP F.U. $\times 10^{5}$ )'. In addition, Supplementary Fig. S6 was incorrect as originally published and was replaced on 26 January 2006. 